\title{
Controle Ótimo da Fermentação Alcoólica em Reator Batelada Alimentada usando Computação Bio-Inspirada
}

\author{
F.S. LOBATO* \\ Recebido em 15 abril, 2013 / Aceito em 24 janeiro, 2015
}

\begin{abstract}
RESUMO. O modo de operação batelada alimentada é reconhecido por amenizar os efeitos de inibição causados pelo produto e pelo substrato no processo de fermentação alcoólica. A otimização dinâmica deste processo para fins de maximização da sua produtividade, através do controle da vazão de alimentação do substrato, caracteriza um problema de controle ótimo singular de difícil solução devido a flutuação do índice diferencial. Neste trabalho, este problema de otimização é resolvido usando o Algoritmo de Colônia de Morcegos, técnica bio-inspirada na natureza que se fundamenta no comportamento social de colônias de morcegos. Os resultados obtidos com este algoritmo são comparados a uma estratégia utilizada para a transformação do problema original em um de identificação de fases de índice diferencial flutuante e de tempos de transição entre elas. Na aplicação proposta são consideradas restrições na produtividade, na variável de controle e no volume do fermentador, para operação com tempo livre. São analisadas o efeito das restrições operacionais e das condições iniciais sobre os perfis ótimos.
\end{abstract}

Palavras-chave: controle ótimo singular, fermentação alcoólica, algoritmo de colônia de morcegos.

\section{INTRODUÇÃO}

Nos últimos anos, os investimentos em processos para a produção de etanol como alternativa aos combustíveis à base de petróleo tem aumentado significativamente devido à razões econômicas e ecológicas. Neste contexto, a modelagem matemática do processo de fermentação batelada alimentada para a produção de etanol a partir de Saccharomyces cerevisae caracteriza um problema de grande interesse na engenharia química. Isto se deve, dentre outros aspectos, aos efeitos inibitórios provocados por altas concentrações de produto e de substrato e por caracterizar um problema de controle ótimo com índice diferencial flutuante [13, 14]. Matematicamente, o com-

Faculdade de Engenharia Química, Universidade Federal de Uberlândia, Campus Santa Mônica, Cx. Postal 593, 38408-100, Uberlândia, MG, Brasil. E-mails: fslobato@feq.ufu.br; franpi22@yahoo.com.br *O autor agradece o suporte financeiro da FAPEMIG e do CNPq. Este trabalho é dedicado à Bianca. 
portamento dinâmico deste processo pode ser representado pelas seguintes equações de balanço de massa $[1,4,7,8]$ :

$$
\begin{gathered}
\frac{d x}{d t}=x \mu-u \frac{x}{V} \quad x(0)=x_{o} \\
\frac{d s}{d t}=-x \frac{\mu}{Y_{x s}}+u\left(\frac{s_{i}-s}{V}\right) \quad s(0)=s_{o} \\
\frac{d p}{d t}=x \varepsilon-u \frac{p}{V} \quad p(0)=p_{o} \\
\mu \frac{\mu_{o}}{1+\frac{p}{K_{p}}} \frac{s}{K_{s}+s} \quad(0 \mathrm{~g} / \mathrm{L} \leq s \leq 120 \mathrm{~g} / \mathrm{L}) \\
\varepsilon=\frac{\varepsilon_{o}}{1+\frac{p}{K_{p 1}}} \frac{s}{K_{s 1}+s} \quad(0 \mathrm{~g} / \mathrm{L} \leq p \leq 80 \mathrm{~g} / \mathrm{L})
\end{gathered}
$$

onde $x, s$ e $p$ são as concentrações de células, substrato e produto, respectivamente. $x_{o}, s_{o}$ e $p_{o}$ representam as concentrações de células, substrato e produto no início do processo (instante de tempo $t$ igual a zero), respectivamente. $u$ é a vazão volumétrica, $s_{i}$ é a concentração da corrente de substrato alimentado, $V$ é o volume do fermentador, $Y_{x s}$ é o coeficiente de rendimento, $\mu$ é a taxa de crescimento específica e $\varepsilon$ é a produtividade específica. Já $K_{s}, K_{s 1}, K_{p}, K_{p 1}, \mu_{o}$ e $\varepsilon_{o}$ são parâmetros cinéticos referentes ao modelo de massa apresentado.

Do ponto de vista de controle, a trajetória ótima da variável de controle que maximiza a produção de etanol $(p V)$ consiste de arcos não singulares e singulares, caracterizando um Problema de Controle Ótimo Singular (PCOS), para o qual a sequência de arcos e os tempos nos quais ocorre a transição entre eles (eventos ou switching times) têm que ser determinados. Na literatura, inúmeras metodologias tem sido propostas para a resolução deste problema. Hong [8], aplicando a transformação de Kelly para diminuir a dimensão do modelo, obteve uma expressão analítica para a condição de conjunção entre os arcos singulares e não singulares, analisando o efeito das condições iniciais sobre a trajetória ótima. Segundo o autor, a pouca precisão das equações cinéticas para $\mu$ e $\varepsilon$ seria responsável pela obtenção do rendimento em produto igual a 0,73 , superior ao rendimento teórico da formação de etanol a partir de glicose $\left(Y_{p s}\right)$ $\left(\mathrm{C}_{6} \mathrm{H}_{12} \mathrm{O}_{6} \rightarrow 2 \mathrm{C}_{2} \mathrm{H}_{6} \mathrm{O}_{2}+2 \mathrm{CO}_{2}\right)$ para o Shaccaromyces cerevisae, que é 0,51. Para concentrações iniciais altas de substrato ( $>100 \mathrm{~g} / \mathrm{L}$ ), o perfil ótimo de $u$ consistiu de uma vazão mínima seguida por um perfil singular. Os eventos foram obtidos pela aplicação do Princípio do Mínimo de Pontryagin [3], como funções das variáveis de estado e dos parâmetros cinéticos. A abordagem utilizada por estes autores obtém a solução do problema singular a partir da solução de um problema semi singular. Modak et al. [17], admitindo que a formação de produto ocorre após o crescimento celular, propuseram uma transformação do vetor de estados que resultou num problema de controle não singular e mostraram que a sequência ótima geral é definida por: máxima 
alimentação, batelada, singular e batelada, e que casos particulares dependem das formas monotônicas ou não monotônicas das taxas específicas de crescimento, de formação de produto e das condições iniciais. Entretanto, segundo Modak \& Lim [18], a incorporação explícita de restrições físicas e a não monotonicidade da taxa específica de crescimento podem limitar a aplicação desta estratégia.

O ponto de vista algébrico-diferencial foi incorporado na obtenção da estratégia de controle on-off por Chen \& Hwang [4], aliado à utilização da transformação de Kelly. Fu \& Barford [7] incorporaram uma restrição na produtividade específica $\varepsilon$ para atender à estequiometria da reação e obtiveram uma solução ótima não singular pela aplicação do princípio de Pontryagin, que resultou num problema de valor no contorno em dois pontos. Com isto, foram evitados valores de concentração de etanol superiores ao teórico possível (76,65 g/L nas condições analisadas). Isto pode ser constatado na Tabela 1, que mostra resultados obtidos por vários autores e na qual fica evidente que, com exceção do caso $\mathrm{E}$, todos os demais não consideram esta limitação física. Por outro lado, para todos eles o tempo de fermentação é excessivo, seja para tempos fixos ou livres.

Tabela 1: Comparação entre soluções ótimas obtidas com e sem restrição na produtividade.

\begin{tabular}{|c|c|c|c|c|c|}
\hline Caso & Tempo $(\mathrm{h})$ & $x(\mathrm{~g} / \mathrm{L})$ & $p(\mathrm{~g} / \mathrm{L})$ & $p V(\mathrm{~g})$ & Referência \\
\hline A & 59,05 & 14,8 & 105,00 & 21000,0 & {$[8]$} \\
B & 54,00 & 14,5 & 100,37 & 20073,2 & \\
C & $50,00^{*}$ & 15,0 & 99,98 & 19996,2 & {$[4]$} \\
D & 56,00 & 15,0 & 105,70 & 21140,2 & \\
E & 50,67 & 15,0 & 75,68 & 15132,0 & {$[7]$} \\
F & $63,00^{*}$ & - & 101,78 & 20355,0 & {$[22]$} \\
\hline
\end{tabular}

Durante décadas, a resolução do PCOS ficou restrita ao uso de métodos determinísticos, a saber, métodos diretos, indiretos e híbridos [3, 6, 11, 15]. Nos últimos anos, o desenvolvimento de novos métodos de otimização, associado à sofisticação dos computadores, tem possibilitado o aprimoramento de estratégias sistemáticas para a resolução do PCOS. Dentre estas estratégias destaca-se o Algoritmo de Colônia de Morcegos (ACM), proposto por Yang [23]. Este algoritmo é conceitualmente baseado no comportamento social de colônias de morcegos. Dentre as características observadas em uma colônia de morcegos pode-se enumerar as seguintes [23, 24]: i) os morcegos emitem sons de vários comprimentos de onda e frequência na busca por presas e para ajudar no seu direcionamento; $i$ ) os morcegos voam com velocidade $v$ com diferentes comprimentos de onda, $\mathrm{iii}$ ) os morcegos ajustam a sua velocidade, direção e frequência ao ouvir sinal de eco. Baseado nestas observações, Yang [23] propôs uma estratégia para atualizar/gerar candidatos em potencial para a solução de problemas de otimização. Na literatura, podem ser encontradas aplicações do ACM, dentre os quais pode-se citar: otimização de funções matemáticas [5, 10, 23, 24], otimização multi-objetivo [25], estimação de parâmetros em sistemas biológicos 
[9], classificação de dados [16], projeto de sistemas de engenharia [26], resolução de problemas de programação de produção [19], e problemas de otimização estocásticos [20].

Diante do que foi apresentado, o presente trabalho tem por objetivo propor uma metodologia sistemática para o tratamento do PCOS usando o ACM. A abordagem proposta é aplicada para a determinação do perfil ótimo da variável de controle no processo de fermentação batelada alimentada com tempo de operação livre, e sujeito a restrições de produtividade, controle e volume do reator. Este trabalho está estruturado como segue: a Seção 2 apresenta a descrição matemática do PCOS; nas Seções 3 e 4 são apresentadas a concepção conceitual do ACM e a metodologia proposta para o tratamento do PCOS; na Seção 5 são apresentados estudos de caso. Finalmente, as conclusões e perspectivas futuras são apresentadas na Seção 6.

\section{FORMULAÇÃO MATEMÁTICA DO PCOS}

A formulação matemática do PCOS genérico é dado por [3]:

$$
\begin{gathered}
\min J=\psi\left(\mathbf{x}\left(t_{f}\right), t_{f}\right)+\int_{t_{0}}^{t_{f}} L(\mathbf{x}, \mathbf{u}, t) d t \\
f(\dot{\mathbf{x}}, \mathbf{x}, \mathbf{u}, t)=0 \\
S(\mathbf{x}, \mathbf{u}, t) \leq 0 \\
\varphi\left(\mathbf{x}\left(t_{f}\right)\right)=0 \\
\mathbf{x}^{L} \leq \mathbf{x} \leq \mathbf{x}^{U} \\
\mathbf{u}^{L} \leq \mathbf{u} \leq \mathbf{u}^{U}
\end{gathered}
$$

onde $\mathbf{x}$ é o vetor das variáveis de estado, $\mathbf{u}$ é o vetor das variáveis de controle, $\psi\left(\mathbf{x}\left(t_{f}\right), t_{f}\right)$ é o primeiro termo da função objetivo avaliado em $t=t_{f}$ e $L(\mathbf{x}, \mathbf{u}, t)$ é o segundo termo do funcional $J, S(\mathbf{x}, \mathbf{u}, t)$ é o vetor de restrições de desigualdade, $f(\dot{\mathbf{x}}, \mathbf{x}, \mathbf{u}, t)$ é o vetor de restrições algébricodiferenciais, e $\varphi\left(\mathbf{x}\left(t_{f}\right)\right)$ é o vetor de variáveis definidas no tempo final $t_{f}$. Os sobrescritos $L$ e $U$ identificam, respectivamente, os limites inferior e superior das variáveis.

Conforme mencionado anteriormente, a resolução do PCOS pode ser obtida via métodos diretos, indiretos e híbridos [3]. No caso dos métodos diretos, estes consistem na discretização do vetor de variáveis de controle e de estado ou apenas do vetor de variáveis de controle. Assim, temse a formulação de problema de programação não linear. Já os métodos indiretos consistem da aplicação do Cálculo Variacional para a dedução das condições necessárias e suficientes para a solução do PCOS [3]. Tal metodologia consiste da transformação do problema original em um problema de valor no contorno algébrico-diferencial.

Seja o problema de controle descrito pelas equações (2.1) e (2.2). Para este sistema, a função Hamiltoniano é definida como [3, 6, 11]:

$$
H \equiv L(\mathbf{x}, \mathbf{u}, t)+\lambda^{T} f
$$


A partir da aplicação do Princípio de Pontriagyn, obtemos as condições de otimalidade ou Equações de Euler-Lagrange [3, 6, 11]:

$$
\begin{aligned}
& \dot{\lambda}=-\frac{\partial H}{\partial \dot{\mathbf{x}}} \quad \lambda\left(t_{f}\right)=\frac{\partial \psi\left(\mathbf{x}\left(t_{f}\right), t_{f}\right)}{\partial \mathbf{x}\left(t_{f}\right)} \\
& \frac{\partial H}{\partial \mathbf{u}}=0
\end{aligned}
$$

onde $\lambda$ é o vetor de variáveis adjuntas (co-estado) e a equação (2.9) é conhecida como condição estacionária.

A Teoria de Controle Ótimo diz que a estratégia de controle ótimo para o PCOS linear com relação à variável de controle deve satisfazer o seguinte critério [3]:

$$
u(t)= \begin{cases}u_{\max } & \text { se } \mathrm{FIF}<0 \\ u_{\text {singular }} & \text { se } \mathrm{FIF}=0 \\ u_{\text {min }} & \text { se } \mathrm{FIF}>0\end{cases}
$$

onde FIF é a Função Identificadora de Fases [11], definida como $\frac{d^{n}}{d t^{n}}\left(\frac{\partial H}{\partial \mathbf{u}}\right)$, onde $n$ é o número de diferenciações necessárias para explicitar a variável de controle. Neste caso, $n+1$ caracteriza o índice diferencial, i.e., o número mínimo de vezes que o sistema algébrico-diferencial ou parte dele deve ser diferenciado com relação ao tempo para determinação de um sistema puramente diferencial [2]. Tal conceito está diretamente relacionado ao grau de dificuldade de resolução de um sistema algébrico-diferencial, decorrente de mal condicionamento, instabilidade, singularidade e má convergência $[3,6,11,15]$.

\section{O ALGORITMO DE COLÔNIA DE MORCEGOS}

O algoritmo proposto por Yang [23] fundamenta-se no processo de eco-localização desempenhada pela colônia de morcegos durante o seu vôo para fins de detecção de presas e para evitar obstáculos. Esta eco-localização baseia-se na emissão de ondas ultrassônicas e a correspondente medição do tempo gasto para que estas ondas voltem à fonte após serem refletidas no alvo (presa ou obstáculo). Do ponto de vista biológico, quando um morcego identifica uma presa, a taxa de pulso $(r)$ é acelerada e a amplitude $(A)$ é aumentada para evitar que a presa escape. Por outro lado, quando a presa está sob o domínio, a amplitude diminui [5].

Em termos práticos, no ACM cada morcego representa uma solução em potencial para o problema de otimização. Assim, uma população de morcegos move-se no espaço de busca do problema, continuamente atualizando a frequência $(f)$, a velocidade e a posição de seus indivíduos (morcegos), objetivando a captura da presa. A cada nova iteração, a posição de cada morcego é atualizada seguindo a melhor solução encontrada pela população (comportamento social). É importante ressaltar que o "controle" de exploração e de intensificação do processo de busca neste algoritmo é realizado via variação da amplitude e da taxa de pulso [5, 23].

O pseudo-código para o ACM é apresentado a seguir [5]. 


\section{Algoritmo de Colônia de Morcegos}

1: Definição dos parâmetros: $F, N P, n_{\text {iter }}, d, \alpha, f_{\min }, f_{\max }, r_{o}$ e $A_{o}$

2: Inicialização da população $(\vec{x})$

3: Avaliação de cada indivíduo da população - função objetivo $(F(\vec{x}))$

4: Atualização do melhor morcego $\left(\vec{x}_{*}\right)$

5: white (o critério de parada não for satisfeito) do

6: $\quad$ for $t$ from 1 to $n_{\text {iter }}$ do

7: $\quad f_{i}=f_{\text {min }}+f_{\text {max }} \beta(\beta \in[0,1])$

8: $\quad \vec{v}_{i}^{t+1}=\vec{v}_{i}^{t}+\left(\vec{x}_{i}^{t}-\vec{x}_{*}^{t}\right) f_{i}$

9: $\quad \vec{x}^{t+1}=\vec{x}^{t}+\vec{v}^{t+1}$

10: $\quad$ if rand $<r_{i}$ (rand $\left.\in[0,1]\right)$ then (busca local)

11: $\quad \vec{x}_{i}^{t}=\vec{x}_{*}^{t}+\rho A_{i}^{t}(\rho \in[-1,1])$

12: $\quad$ end if

13: $\quad$ Perturbação da $i$-ésima posição $\vec{x}$

14: $\quad$ if rand $<A_{i}$ or $F\left(\vec{x}_{i}\right) \leq F\left(\vec{x}_{*}\right)$ then

15: $\quad \vec{x}_{*}=\vec{x}_{i}$

16: $\quad r_{i}^{t+1}=1-\exp (-\rho t)$

17: $\quad A_{i}^{t+1}=\alpha A_{i}^{t}$

18: $\quad$ end if

19: Atualiza o melhor candidato $\vec{x}_{*}$

\section{0: end for}

\section{1: end while}

22: Pós-processamento

Este processo é inicializado escolhendo-se aleatoriamente a posição e a velocidade da população de morcegos $\left(\vec{x}_{i} ; i=1, \ldots, N P\right)$, onde $N P$ é o tamanho da população. Considera-se inicialmente a amplitude $A_{i}=1$ e a frequência $f_{i}=0$ (linha 2). O ciclo principal representa a evolução da população com o tempo (linhas 5 à 21). O primeiro passo é atualizar a posição temporária $\vec{x}_{i}$ até esta ser aceita. Para isto, a frequência $f_{i}$ é atualizada (linha 7), onde $f_{\min }$ e $f_{\text {max }}$ são os limites inferiores e superiores da frequência, respectivamente e que correspondem a uma determinada faixa de comprimento de onda. $\beta$ é um número aleatório pertencente ao intervalo [0,1]. Neste caso, a nova frequência $f_{i}$ é utilizada para determinar a nova velocidade $\vec{v}_{i}^{t+1}$ (linha 8), onde $\vec{x}_{*}$ é a melhor solução na geração corrente. De posse da nova velocidade $\vec{v}_{i}^{t+1}$, é possível determinar a nova posição (linha 9). Na linha 10 é realizada uma busca local. Na linha 13, a $i$-ésima posição $\vec{x}_{i}$ é escolhida aleatoriamente para ser perturbada considerando o domínio das variáveis de projeto. Se a condição dada na linha 14 for satisfeita a solução temporária $\vec{x}_{i}$ é aceita (linha 15) e também ocorre o aumento da taxa de pulso (linha 16), sendo que quando $t \rightarrow \infty$ e $r_{i} \rightarrow 1$, a busca local se intensifica. $\mathrm{O}$ valor da amplitude $A$ é atualizado na linha 17. Neste caso, a $A$ é reduzida a uma taxa através da relação $A=A_{o} \alpha^{t},\left(t=1, \ldots, n_{\text {iter }}\right)$ e $0<\alpha<1$. O processo continua até o número máximo de iterações $\left(n_{\text {iter }}\right)$ ser alcançado. 
É importante ressaltar que outros critérios para a finalização do processo iterativo podem ser empregados, dentre os quais pode-se citar: $i$ ) o número máximo de avaliações da função objetivo, ii) intervenção humana e iii) tempo de processamento. Todavia, a escolha do número máximo de iterações como critério de parada é a forma usual empregada para essa finalidade, sendo que o valor encontrado é comparado com um valor de referência descrito na literatura especializada, o que assegura a qualidade da solução encontrada pela metodologia proposta para o número de iterações considerado. Caso este conjunto de parâmetros não seja suficiente para a obtenção de uma solução satisfatória, deve-se modificar os parâmetros do algoritmo, dentre os quais podese avaliar o efeito do número máximo de iterações. Finalmente, ressalta-se que nenhum dos critérios citados garantem que a solução ótima foi encontrada, mas o uso de uma solução de referência associado com a análise de sensibilidade dos parâmetros do algoritmo pode dar um bom indicativo da qualidade da solução obtida.

\section{METODOLOGIA}

O PCOS pode ser reescrito como um problema definido por $m$ elementos de controle (fases), que são conectadas por eventos $\left(t s_{i}, i=1, \ldots, m-1\right)$ para um tempo final fixo ou não [11]. A Figura 1 ilustra bem essa ideia.

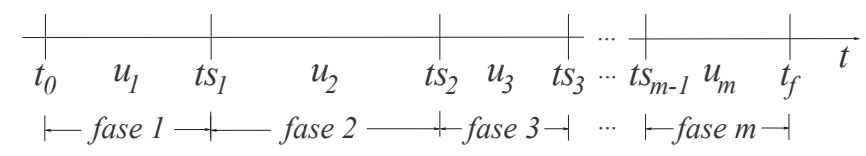

Figura 1: PCOS definido em elementos de controle.

Baseando-se nesta figura, a metodologia proposta neste trabalho consiste no uso do ACM para a determinação da vazão de alimentação de substrato (variável de controle $u$ ) de modo a maximizar a produção de etanol $p V$ ao final do tempo de operação. De forma resumida, a metodologia é fundamentada nos seguints passos:

- definir o número de elementos de controle $(m)$, de tamanho variável, para as quais, em cada um destes elementos, a variável de controle é $u \equiv u_{i}$, onde $u_{i}$ é a $i$-ésima variável de controle $(i=1, \ldots, m)$, bem como os parâmetros do ACM;

- aplicar o ACM para a determinação de $u_{i}$ e do tamanho do elemento de controle $\left(t s_{i+1}-\right.$ $\left.t s_{i}, i=1, \ldots, m-1\right)$. Para problemas com tempo final livre, $t_{f}$ passa a ser uma variável a ser determinada pela metodologia.

Neste caso, como o valor da função objetivo $(F)$ é dependente da escolha do vetor de variáveis de projeto, a cada execução do ACM, um novo vetor de candidatos à solução do problema de otimização é gerado. Assim, de posse deste vetor, o sistema algébrico-diferencial é resolvido através do uso da aplicação do Método de Runge-Kutta-Fehlberg e o valor da função objetivo é 
obtida. Este processo é repetido até que um determinado critério de parada seja satisfeito. Neste trabalho adotou-se como critério de parada o número máximo de iterações.

Para fins de comparação, os resultados obtidos com a aplicação do ACM via discretização do vetor de variáveis de controle serão confrontados com os obtidos pela metodologia proposta por Lobato [12]. Neste caso, a partir da definição de uma nova variável de controle, a saber, a taxa de diluição $(D \equiv u / V)$, a dimensão do problema original é reduzida em uma unidade através da eliminação estratégica da equação do balanço global $(\dot{V}=u)$. Lobato [12] demonstrou a partir de manipulações algébricas que, para o sistema formado pelas equações (1.1)-(1.6), é possível obter uma estratégia ótima de controle que é função apenas do vetor de variáveis de estado, i.e., que não depende do vetor de variáveis adjuntas, e que o índice diferencial deste sistema é 3. Mais detalhes sobre o desenvolvimento matemático podem ser encontrados em [12].

\section{RESULTADOS E DISCUSSÃO}

Nesta seção são apresentados cinco estudos de caso para a validação da metodologia proposta. Para essa finalidade alguns pontos devem ser destacados:

- Parâmetros [1, 8]: $K_{s}=0,22 \mathrm{~g} / \mathrm{L} ; K_{s 1}=0,44 \mathrm{~g} / \mathrm{L} ; K_{p}=16,0 \mathrm{~g} / \mathrm{L} ; K_{p 1}=71,5 \mathrm{~g} / \mathrm{L}$; $\mu_{o}=0,408 \mathrm{~h}^{-1} ; \varepsilon_{o}=1,0 \mathrm{~h}^{-1}, Y_{x s}=0,1 ; x_{o}=1 \mathrm{~g} / \mathrm{L}, p_{o}=0 \mathrm{~g} / \mathrm{L} \mathrm{e} s_{i}=150 \mathrm{~g} / \mathrm{L} . \mathrm{Em}$ todas as simulações foram utilizados 40 elementos de controle;

- Parâmetros do ACM [5, 23]: $N P=50 ; n_{\text {iter }}=500 ; \alpha=0,9 ; f_{\min }=0 ; f_{\max }=2 ; r_{o}=$ 0,5 e $A_{o}=0,25$. Para estes parâmetros são necessárias $50+50 \times 500$ avaliações da função objetivo. O algoritmo foi executado 10 vezes para a apresentação dos resultados;

- Função Objetivo $(F)$ : maximização do produto $p V$ (ou minimizar - $p V$ ), sujeito às equações (1.1)-(1.6), com tempo final livre, volume final do reator igual a 200 L, com a variável de controle limitada por $u_{\min }=0 \mathrm{~L} / \mathrm{h}$ e $u_{\max }=12 \mathrm{~L} / \mathrm{h}, p V \in[0 \infty[$, e restrição no rendimento [21]:

$$
\frac{p(t) V(t)-p_{o} V_{o}}{\left(V(t)-V_{o}\right) s_{i}+V_{o} s_{o}-V(t) s(t)} \leq 0,51
$$

- Como $\mu$ (equação (1.5)) é não monotônica e a concentração de substrato é alta, a vazão de substrato alimentado inicialmente deve ser mínima, o que no limite ocorre no modo batelada, até que o substrato em excesso seja consumido e o arco singular seja alcançado;

- Na fase batelada ( $u=u_{\text {min }}$, índice diferencial igual a 1), o sistema de equações diferenciais ordinárias é resolvido a partir de $t=t_{o}$ até que a singularidade seja identificada, o que ocorre em $t=t_{I}$. Neste evento inicia-se a fase singular ( $u=u_{\text {singular }}$, índice diferencial igual a 3). Em $t=t_{I I}$ a restrição no rendimento é ativada até que a variável de controle alcance o valor máximo em $t=t_{I I I}\left(u=u_{\max }\right.$, índice diferencial igual a 1). Quando o reator é totalmente preenchido, o que ocorre em $t=t_{I V}$, a alimentação é interrompida e $u$ atinge seu limite inferior $\left(u=u_{m i n}\right.$, índice diferencial igual a 1$)$. 
A Tabela 2 apresenta os melhores resultados obtidos para os eventos calculados para diferentes volumes iniciais do reator utilizando a metodologia proposta por Lobato [12] e pelo ACM. Nesta tabela é possível observar boa concordância entre os resultados obtidos pela metodologia proposta por Lobato [12] e pelo ACM, com desvios da ordem de $0,01 \%$ à $0,03 \%$.

Tabela 2: Condições iniciais, eventos e função objetivo para os casos estudados.

\begin{tabular}{|c|c|c|c|c|c|c|c|c|c|}
\hline & Caso & $V_{o}(\mathrm{~L})$ & $s_{o}(\mathrm{~g} / \mathrm{L})$ & $t_{I}(\mathrm{~h})$ & $t_{I I}(\mathrm{~h})$ & $t_{I I I}(\mathrm{~h})$ & $t_{I V}(\mathrm{~h})$ & $t_{f}(\mathrm{~h})$ & $p V(\mathrm{~g})$ \\
\hline$[12]$ & \multirow{2}{*}{1} & \multirow{2}{*}{10} & \multirow{2}{*}{150,0} & 13,002 & 17,124 & 50,993 & 53,750 & 54,706 & 15229 \\
$\mathrm{ACM}$ & & & & 13,012 & 17,128 & 50,995 & 53,754 & 54,701 & 15226 \\
\hline$[12]$ & \multirow{2}{*}{2} & \multirow{2}{*}{50} & \multirow{2}{*}{120,0} & 10,622 & 17,850 & 26,275 & 29,177 & 30,044 & 14534 \\
$\mathrm{ACM}$ & & & & 10,620 & 17,843 & 26,222 & 29,157 & 30,001 & 14531 \\
\hline$[12]$ & \multirow{2}{*}{3} & \multirow{2}{*}{100} & \multirow{2}{*}{135,0} & 11,802 & 17,431 & 17,733 & 21,007 & 21,851 & 14535 \\
$\mathrm{ACM}$ & & & & 11,799 & 17,429 & 17,730 & 21,002 & 21,850 & 14534 \\
\hline$[12]$ & \multirow{2}{*}{4} & \multirow{2}{*}{145,63} & \multirow{2}{*}{139,2} & 12,138 & 17,202 & - & - & 17,790 & 14450 \\
$\mathrm{ACM}$ & & & & 12,119 & 17,200 & - & - & 17,787 & 14449 \\
\hline$[12]$ & \multirow{2}{*}{5} & \multirow{2}{*}{200} & \multirow{2}{*}{142,5} & - & - & - & - & 13,321 & 12960 \\
$\mathrm{ACM}$ & & & & - & - & - & - & 13,313 & 12957 \\
\hline
\end{tabular}

O Caso 1 é o caso base, no qual as concentrações de substrato alimentado $\left(s_{i}\right)$ e inicial $\left(s_{o}\right)$ são iguais e o volume inicial do fermentador é de $10 \mathrm{~L}$. É o caso em que se alcança a maior produtividade às custas de um grande tempo de fermentação, a maior parte dele $\left(t_{I I}<t<t_{I V}\right)$ consumido no enchimento do reator sem alterações significativas na concentração de produto. No início da fase singular $\left(t=t_{I I}\right)$ ocorre um ligeiro decréscimo na concentração de células, coincidente com a concentração de substrato praticamente constante. Esta tendência se reverte na última fase $\left(t_{I V}<t<t_{f}\right)$ quando a operação volta a ser batelada e o decréscimo da concentração de substrato ocorre simultaneamente ao crescimento celular e à formação de produto. Como do ponto de vista prático este tempo de fermentação é considerado excessivo, nos Casos 2 e 3 foram simuladas situações para as quais os volumes e as concentrações iniciais de substrato e o tempo de operação batelada inicial são crescentes, enquanto as respectivas fases singulares sofrem reduções, i.e., o tempo de operação semi-contínua diminui. Embora as produtividades sejam da ordem de 4,56\% e 4,55\% inferiores à do Caso 1, os tempos de fermentação sofrem uma significativa redução de $45,08 \%$ e $60,05 \%$, respectivamente.

O efeito de um controle bang-bang foi analisado no Caso 4, onde a trajetória ótima consiste de $u_{\min }-u_{\max }-u_{\min }$, estando o reator de $200 \mathrm{~L}$ preenchido inicialmente com 145,63 L. Com a eliminação da fase singular, o tempo de fermentação foi reduzido de 54,706 h do caso base para 17,79 h, o que representa $67,48 \%$, com uma redução pouco significativa na produtividade.

Estas análises mostram que no caso analisado, a desejada redução do tempo de fermentação pode ser obtida às custas da eliminação do modo de alimentação semi-contínuo, fazendo crer que a operação batelada seria mais conveniente para este processo. A operação batelada também foi 
simulada e apresentada como o Caso 5. Fica evidente que, mesmo conseguindo maior redução no tempo de fermentação, a redução no valor da função objetivo passa a ser bem mais significativa com relação aos Casos 2, 3 e 4.

Nas Figuras 2 e 3 são apresentados os perfis de concentração de células, substrato, etanol, volume e controle para cada um dos estudos de caso obtidos pelo ACM.

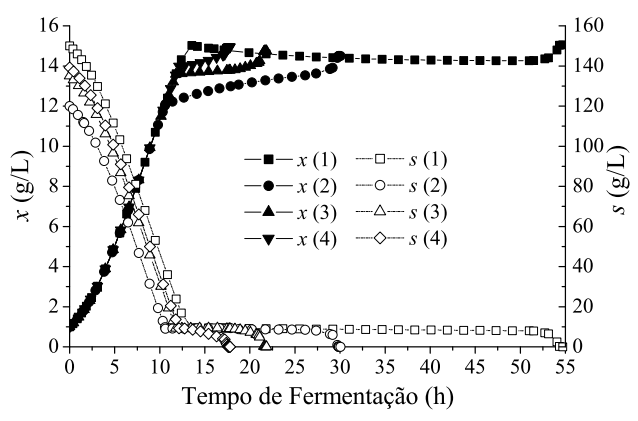

(a) Perfil ótimo de células e substrato.

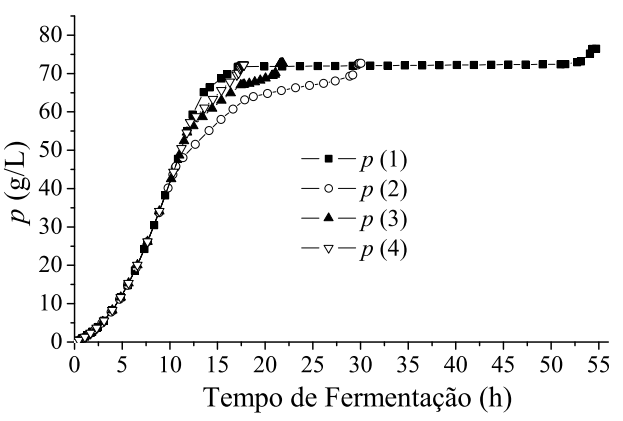

(b) Perfil ótimo de etanol.

Figura 2: Perfis das variáveis de estado $x, s$ e $p$ para os estudos de caso propostos.

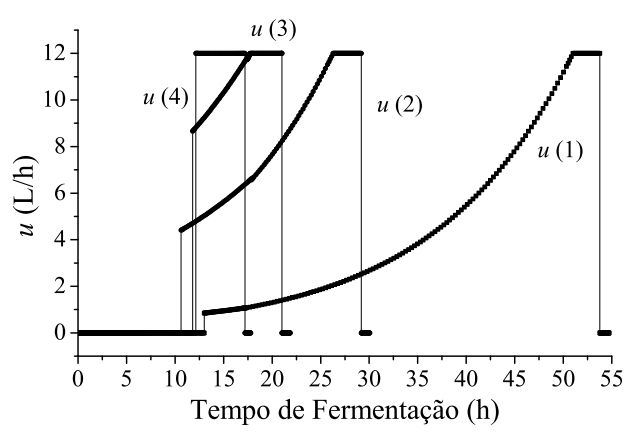

(a) Controle ótimo.

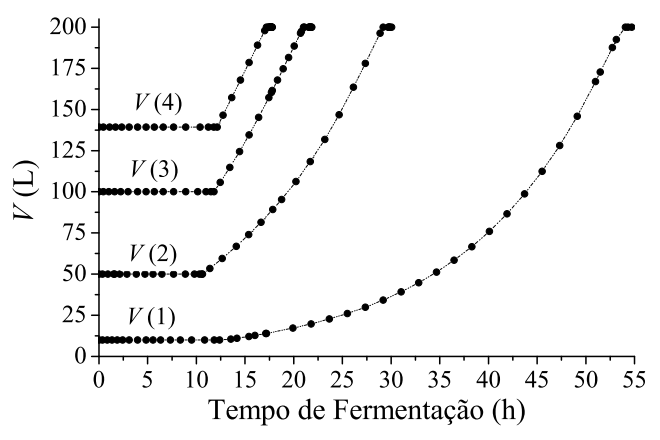

(b) Volume.

Figura 3: Perfis das variáveis de estado $V$ e de controle $u$ para os estudos de caso propostos.

A Tabela 3 apresenta o desvio padrão considerando diferentes volumes iniciais do reator para as dez execuções do algoritmo.

A partir da análise dos resultados apresentados nesta tabela é possível observar que o ACM foi capaz de obter resultados satisfatórios devido a magnitude dos valores do desvio padrão referente 
Tabela 3: Desvio padrão para os casos estudados.

\begin{tabular}{|c|c|c|c|c|c|c|c|c|}
\hline Caso & $V_{o}(\mathrm{~L})$ & $s_{o}(\mathrm{~g} / \mathrm{L})$ & $t_{I}(\mathrm{~h})$ & $t_{I I}(\mathrm{~h})$ & $t_{I I I}(\mathrm{~h})$ & $t_{I V}(\mathrm{~h})$ & $t_{f}(\mathrm{~h})$ & $p V(\mathrm{~g})$ \\
\hline 1 & 10 & 150,0 & 0,065 & 0,0123 & 0,0736 & 0,1098 & 0,006 & 129 \\
\hline 2 & 50 & 120,0 & 0,282 & 0,599 & 0,798 & 0,097 & 0,033 & 145 \\
\hline 3 & 100 & 135,0 & 0,626 & 0,198 & 0,303 & 0,097 & 0,591 & 149 \\
\hline 4 & 145,63 & 139,2 & 0,778 & 0,098 & - & - & 0,990 & 178 \\
\hline 5 & 200 & 142,5 & - & - & - & - & 0,271 & 132 \\
\hline
\end{tabular}

ao vetor de variáveis de projeto. Em termos do valor da função objetivo, os valores obtidos também são considerados satisfatórios em relação aos obtidos por Lobato [12].

\title{
6 CONCLUSÕES
}

O presente trabalho propôs uma metodologia para a redução da fase singular do problema de controle ótimo da fermentação do tipo batelada alimentada para produção de etanol a partir de glicose através do Saccharomyces cerevisae. Para essa finalidade, foi avaliada a influência do volume inicial e, por consequência, da concentração inicial de substrato no fermentador. De posse dos resultados obtidos observa-se que houve uma redução significativa do tempo de fermentação mas sem que exista uma diminuição proporcional na produtividade alcançada. A metodologia de solução empregada, em que modelos de valor inicial são resolvidos a cada fase, cujas características são definidas pelos respectivos índices e que necessitam da determinação dos vários eventos através do ACM apresentou bons resultados, comparáveis aos descritos na literatura e pela metodologia proposta por Lobato [12]. Como sequência deste trabalho pretende-se avaliar o desempenho do ACM com relação aos seus parâmetros e a sua extensão para o contexto multi-objetivo.

\begin{abstract}
The fed-batch operation is recognized by soften the inhibition effects caused by product and substrate in alcoholic fermentation process. The dynamic optimization of this process for purposes of productivity maximization, through control of substrate feed flow, characterizes a singular optimal control problem of difficult solution due to differential index fluctuation. In this work, this optimization problem is solved using the Bat Colony Algorithm, bio-inspired technique in nature that is based on social behavior of bats colonies. The results obtained with this algorithm are compared to a strategy used for transformation of original problem in one of phases identification. In application proposed are considered constraints of productivity, control variable and volume, for operation with free time.
\end{abstract}

Keywords: singular optimal control, alcoholic fermentation process, bat colony algorithm. 


\section{REFERÊNCIAS}

[1] S. Aiba, M. Shoda \& M. Nagatani. Kinetics of product inhibition in alcohol fermentation. Biotechnology and Bioengineering, X(6) (1968), 845-864.

[2] K.E. Brenan, S.L. Campbell \& L.R. Petzold. "Numerical Solution of Initial Value Problems in Differential Algebraic Equations”. Classics in Applied Mathematics, SIAM Philadelphia, (1996).

[3] A.E. Bryson \& Y.C. Ho. "Applied Optimal Control". Hemisphere Publishing, Washington, (1975).

[4] C. Chen \& C. Hwang. Optimal on-off control for fed-batch fermentation processes. Ind. Eng. Chem. Research, 29 (1990), 1869-1875.

[5] J. Cordeiro, R.S. Parpinelli \& H.S. Lopes. Análise de Sensibilidade dos Parâmetros do Bat Algorithm e Comparação de Desempenho. Encontro Nacional de Inteligência Artificial (ENIA), 1 (2012), 1-9.

[6] W.F. Feehery. "Dynamic Optimization with Path Constraints". Thesis, Massachusetts Institute of Technology, (1998).

[7] P.C. Fu \& J.P. Barford. Non-singular optimal control for fed-batch fermentation processes with a differential-algebraic system model. J. Proc. Control, 12 (1993), 211-218.

[8] J. Hong. Optimal substrate feeding policy for a fed batch fermentation with substrate and product inhibition kinetics. Biotechnology and Bioengineering, XXVIII (1986), 1421-1431.

[9] J.H. Lin, C.W. Chou, C.H. Yang \& H.L. Tsai. A Chaotic Levy Flight Bat Algorithm for Parameter Estimation in Nonlinear Dynamic Biological Systems. Journal of Computer and Information Technology, 2(2) (2012).

[10] J. Xie, Y. Zhou \& Huan Chen. A Novel Bat Algorithm Based on Differential Operator and Lévy Flights Trajectory. Computational Intelligence and Neuroscience, 2013 (2013), 1-13.

[11] F.S. Lobato. "Abordagem Mista para Problemas de Otimização Dinâmica". Dissertação de Mestrado, Faculdade de Engenharia Química, Universidade Federal de Uberlândia, (2004).

[12] F.S. Lobato. Determinação do Perfil Ótimo de Alimentação de Substrato no Processo de Fermentação ao Alcoólica - Influência da Condição Inicial. TEMA. Tendências em Matemática Aplicada e Computacional, 12 (2011), 1-10.

[13] F.S. Lobato, K.G. Santos \& V.V. Murata. Controle on-off de um Fermentador Batelada Alimentada para Altas Concentrações de Substrato. 6 Congresso Brasileiro de Engenharia Química em Iniciação Cientifica (COBEQ-IC) (2005).

[14] F.S. Lobato, K.G. Santos, L.C. Oliveira-Lopes \& V.V. Murata. Controle Ótimo da Fermentação Alcoólica com Altas Concentrações Iniciais de Substrato em Reator Batelada Alimentada. XV Simpósio Nacional de Bioprocessos - SINAFERM, Recife - PE. (2005).

[15] J.S. Logsdon \& L.T. Biegler. Accurate Solution of Diferential-Algebraic Optimization Problems. Ind. Eng. Chem. Res., 28 (1989), 89-101.

[16] S. Mishra, K. Shaw \& D. Mishra. A New Meta-heuristic Bat Inspired Classification Approach for Microarray Data. Procedia Technology, 4 (2012), 802-806.

[17] J.M. Modak, H.C. Lim \& Y.J. Tayeb. General characteristics of optimal feed rate profiles for various fed-batch fermentation processes. Biotechnology and Bioengineering, XXVIII (1986), 1396-1407.

[18] J.M. Modak \& H.C. Lim. Simple nonsingular control approach to fed-batch fermentation optimization. Biotechnology and Bioengineering, 33 (1989), 11-15. 
[19] P. Musikapun \& P. Pongcharoen. Solving Multi-Stage Multi-Machine Multi-Product Scheduling Problem Using Bat Algorithm. 2nd International Conference on Management and Artificial Intelligence, 35 (2012).

[20] R. Vanga, M.K. Gupta \& J. Venkateswaran. Performance Evaluation of Bat Algorithm to Solve Deterministic and Stochastic Optimization Problems. International Simulation Conference of India, (2013).

[21] F.S. Wang \& C.H. Shyu. Optimal feed policy for fed-batch fermentation of ethanol production by Zymomous mobilis. Bioprocess Engineering, X (1997), 63-68.

[22] Z. Xiong \& J. Zhang. Modelling and optimal control of fed-batch processes using a novel control affine feedforward neural network. Proceedings of the 2002 American Control Conference, 2 (2002), 5025-5030.

[23] X.S. Yang. "A New Metaheuristic Bat-inspired Algorithm", in: Nature Inspired Cooperative Strategies for Optimization (NISCO 2010) (Eds. J.R. Gonzalez et al.). Studies in Computational Intelligence, Springer, 284, 65-74, (2010).

[24] X.S. Yang. "Nature-Inspired Metaheuristic Algorithms". Second Edition, Luniver Presss, Frome, UK. (2010).

[25] X.S. Yang. Bat Algorithm for Multiobjective Optimization. Int. J. Bio-Inspired Computation, 3(5) (2011), 267-274.

[26] X.S. Yang \& A.H. Gandomi. Bat algorithm: A novel approach for global engineering optimization. Engineering Computations, 29(5) (2012), 464-483. 